\title{
ON PERIODIC TRAVELING WAVE SOLUTIONS OF BOUSSINESQ EQUATION*
}

\author{
BY \\ LIU BAO-PING (Sichuan University and North Carolina State University) \\ AND \\ C. V. PAO (North Carolina State University)
}

\begin{abstract}
This paper is concerned with periodic traveling wave solutions of a generalized Boussinesq equation in the form $u_{t t}=\alpha u_{x x x x}+\left(f_{0}(u)\right)_{x x}$. The basic approach to this problem is to establish an equivalence relation between a corresponding periodic boundary value problem for the traveling wave solution and a Hammerstein type integral equation. This integral representation generates a compact operator in the space of continuous periodic functions of the given period. It is shown by restricting the integral operator on a suitable domain that the Boussinesq equation has the trivial solution as well as a nonconstant periodic traveling wave solution. Special attention is given to the traditional Boussinesq equation where $f_{0}(u)=a u^{2}$. Both of the so called "good" and "bad" Boussinesq equation are treated and the existence of nonconstant traveling wave solution is discussed.
\end{abstract}

1. Introduction. The motion of long waves in shallow water under gravity and in one-dimensional lattices can be described by the Boussinesq equation which is given in the form (cf. [1-7])

$$
u_{t t}=\alpha u_{x x x x}+\left(f_{0}(u)\right)_{x x} \quad\left((t, x) \in \mathbf{R}^{2}\right)
$$

where $\alpha \neq 0$ is a constant and $f_{0}(u)$ is a twice continuously differentiable function of $u$. Eq. (1.0) is often referred to as "good" Boussinesq equation when $\alpha<0$ and "bad" Boussinesq equation when $\alpha>0$. Of special interest is the traditional Boussinesq equation where $f_{0}(u)$ is in the form of a $u^{2}$ for some constant $a>0$ (cf. $[2,3,4,6]$ ). However most of the discussions in the current literature are concerned with soliton solution in the form $v(\xi) \equiv u(x-\beta t)$ to which it is required that $v(\xi) \rightarrow 0$ as $\xi \rightarrow \pm \infty$. In this paper, we investigate periodic traveling wave solution in the same form $v(\xi)=u(x-\beta t)$ for an arbitrary function $f_{0}(u)$, where $\xi \equiv x-\beta t$ and $\beta>0$ is the propagation speed. In terms of $v(\xi)$, Eq. (1.0) is reduced to

$$
c v_{\xi \xi}=v_{\xi \xi \xi \xi}+\alpha^{-1}\left(f_{0}(v)\right)_{\xi \xi}
$$

\footnotetext{
${ }^{*}$ Received by the editors July $28,1983$.
} 
where $c=\beta^{2} / \alpha$. Our aim is to seek a solution of (1.1) subject to the periodicity condition

$$
\frac{d^{k} v}{d \xi^{k}}(0)=\frac{d^{k} v}{d \xi^{k}}(2 T) \text { for } k=0,1,2,3
$$

and the requirement

$$
\int_{0}^{2 T} v(\xi) d \xi=0
$$

where $T>0$ is a preassigned constant. The inclusion of condition (1.3) is to rule out the nontrivial constant solutions of Eqs. (1.1)-(1.2). Throughout the discussion of this paper the period $2 T$ is taken as fixed.

In order to study the boundary-value problem (1.1)-(1.3) we first construct a suitable kernel $K(\xi, \eta)$ and reduce the problem into a Hammerstein type integral equation in the form

$$
v(\xi)=\int_{0}^{2 T} K(\xi, \eta) f(v(\eta)) d \eta \quad(\xi \in[0,2 T])
$$

where $f(v)=|\alpha|^{-1} f_{0}(v)$. In fact, the kernel $K$ is given in terms of explicit trigonometric functions. This elementary property is very useful in justifying that the integral equation (1.4) is a true representation of the boundary-value problem (1.1)-(1.3). Based on the equivalence relation of these two problems we then establish the existence of nontrivial periodic traveling wave solution of the Boussinesq equation via the integral equation (1.4). Special attention will be given to both good and bad Boussinesq equation when $f_{0}(u)=a u^{2}$.

2. An integral representation. In this section we establish an equivalence relation between an integral equation and the boundary-value problem (1.1)-(1.3). The integral representation is based on the explicit construction of a kernel $K(\xi, \eta)$ which is a modified version of a suitable Green's function. This kernel depends on the sign of $\alpha$ so that the "good" and "bad" Boussinesq equation have the same form of integral representation (1.4) but with a different kernel $K$. In fact, by letting

$$
\lambda \equiv|c|^{1 / 2} \equiv \beta|\alpha|^{-1 / 2}
$$

the function $K$ is given according to the sign of $\alpha$ as follows:

(a) For $\alpha>0$ :

$$
K(\xi, \eta)= \begin{cases}(2 \lambda \sinh \lambda T)^{-1} \cosh \lambda(T-\xi+\eta)-\left(2 \lambda^{2} T\right)^{-1}, & 0 \leqslant \eta \leqslant \xi \leqslant 2 T, \\ (2 \lambda \sinh \lambda T)^{-1} \cosh \lambda(-T-\xi+\eta)-\left(2 \lambda^{2} T\right)^{-1}, & 0 \leqslant \xi \leqslant \eta \leqslant 2 T .\end{cases}
$$

(b) For $\alpha<0$ :

$$
K(\xi, \eta)=\left\{\begin{array}{r}
(2 \lambda \sin \lambda T)^{-1} \cos \lambda(T-\xi+\eta)-\left(2 \lambda^{2} T\right)^{-1} \\
0 \leqslant \eta \leqslant \xi \leqslant 2 T \\
(2 \lambda \sin \lambda T)^{-1} \cos \lambda(-T-\xi+\eta)-\left(2 \lambda^{2} T\right)^{-1} \\
0 \leqslant \xi \leqslant \eta \leqslant 2 T
\end{array} \quad(\lambda T \neq n \pi) .\right.
$$


In the above definition of $K$ it is always assumed that $\lambda T \neq n \pi$ for any positive integer $n$ when $\alpha<0$. It is easily seen from (2.1)-(2.2) that $K(\xi, \eta)=K(\eta, \xi)=K(\xi+\delta, \eta+\delta)$ for all $\xi, \eta \in[0,2 T], \delta \in \mathbf{R}$, and thus $K$ is a symmetric kernel for both $\alpha>0$ and $\alpha<0$. In the construction of $K(\xi, \eta)$ we first integrate Eq. (1.1) twice and use the conditions $(1.2)-(1.3)$ to obtain the equation

$$
c v=v_{\xi \xi}+\alpha^{-1}\left[f_{0}(v)-\int_{0}^{2 T} f_{0}(v(\eta)) d \eta\right] .
$$

We then determine the Green's function for the operator $L \equiv\left(d^{2} / d \xi^{2}-\beta^{2} / \alpha\right)$ under the boundary condition (1.2). A modification of Green's function, using conditions (1.2)-(1.3), leads to the form of $K$ on (2.1)-(2.2). In terms of this kernel we have the following representation theorem for (1.1)-(1.3).

THEOREM 1. A continuous function $v(\xi)$ is a solution of the boundary-value problem (1.1)-(1.3) if and only if it is a solution of the integral equation (1.4).

Proof. Define

$$
\begin{aligned}
& G_{1}(z)=(2 \lambda \sinh \lambda T)^{-1} \cosh \lambda(T-z), \\
& G_{2}(z)=(2 \lambda \sin \lambda T)^{-1} \cos \lambda(T-z) .
\end{aligned}
$$

Then the integral equation (1.4) is equivalent to

$$
\begin{aligned}
v(\xi)= & \int_{0}^{\xi} G_{1}(\xi-\eta) f(v(\eta)) d \eta+\int_{\xi}^{2 T} G_{1}(2 T+\xi-\eta) f(v(\eta)) d \eta \\
& -\left(2 \lambda^{2} T\right)^{-1} \int_{0}^{2 T} f(v(\eta)) d \eta,
\end{aligned}
$$

when $\alpha>0$, and it becomes

$$
\begin{aligned}
v(\xi)= & \int_{0}^{\xi} G_{2}(\xi-\eta) f(v(\eta)) d \eta+\int_{\xi}^{2 T} G_{2}(2 T+\xi-\eta) f(v(\eta)) d \eta \\
& -\left(2 \lambda^{2} T\right)^{-1} \int_{0}^{2 T} f(v(\eta)) d \eta
\end{aligned}
$$

when $\alpha<0$. We prove the result for the case $\alpha>0$.

Let $v(\xi)$ be a continuous solution of the integral equation (2.4). It is easily seen from the definition of $G_{1}(z)$ that $G_{1}(0)=G_{1}(2 T)$ and $v(\xi)$ is twice continuously differentiable in $[0,2 T]$. Direct differentiation of $(2.4)$ gives

$$
\begin{aligned}
v^{\prime}(\xi)= & -\lambda C\left[\int_{0}^{\xi} \sinh \lambda(T-\xi+\eta) f(v(\eta)) d \eta+\int_{\xi}^{2 T} \sinh \lambda(-T-\xi+\eta) f(v(\eta)) d \eta\right] \\
v^{\prime \prime}(\xi)= & \lambda^{2} C\left[\int_{0}^{\xi} \cosh \lambda(T-\xi+\eta) f(v(\eta)) d \eta+\int_{\xi}^{2 T} \cosh (-T-\xi+\eta) f(v(\eta)) d \eta\right] \\
& -\lambda C[\sinh \lambda T f(v(\xi))-\sinh (-\lambda T) f(v(\xi))] \\
= & \lambda^{2} v(\xi)+(2 T)^{-1} \int_{0}^{2 T} f(v(\eta)) d \eta-f(v(\xi))
\end{aligned}
$$


where $C=(2 \lambda \sinh \lambda T)^{-1}$. It follows from (2.7) and since $f$ is twice differentiable that $v(\xi)$ is four times differentiable and

$$
v^{\prime \prime \prime \prime}(\xi)=\lambda^{2} v^{\prime \prime}(\xi)-(f(v(\xi)))^{\prime \prime} \quad\left({ }^{\prime} \equiv d / d \xi\right) .
$$

This shows that $v$ is a solution of (1.1). To verify the boundary condition (1.2) we first use the relations (2.4)-(2.6) to obtain

$$
\begin{gathered}
v(0)=\int_{0}^{2 T} G_{1}(2 T-\eta) f(v(\eta)) d \eta-\left(2 \lambda^{2} T\right)^{-1} \int_{0}^{2 T} f(v(\eta)) d \eta=v(2 T), \\
v^{\prime}(0)=-\lambda C \int_{0}^{2 T} \sinh \lambda(-T+\eta) f(v(\eta)) d \eta=v^{\prime}(2 T) .
\end{gathered}
$$

In view of (2.7) we also have $v^{\prime \prime}(0)=v^{\prime \prime}(2 T)$. It follows by differentiating (2.7) and using the above relations that $v^{\prime \prime \prime}(0)=v^{\prime \prime \prime}(2 T)$ and $v^{\prime \prime \prime \prime}(0)=v^{\prime \prime \prime \prime}(2 T)$. To show the relation (1.3) we observe from the Dirichlet integral formula that

$$
\begin{array}{r}
\int_{0}^{2 T} d \xi \int_{0}^{\xi} G_{1}(\xi-\eta) f(v(\eta)) d \eta=\int_{0}^{2 T} f(v(\eta))\left(\int_{\eta}^{2 T} G_{1}(\xi-\eta) d \xi\right) d \eta \\
=\lambda^{-1} C \int_{0}^{2 T} f(v(\eta))[\sinh \lambda T-\sinh \lambda(-T+\eta)] d \eta \\
\int_{0}^{2 T} d \xi \int_{\xi}^{2 T} G_{1}(2 T+\xi-\eta) f(v(\eta)) d \eta=\int_{0}^{2 T} f(v(\eta))\left(\int_{0}^{\eta} G_{1}(2 T+\xi-\eta) d \xi\right) d \eta \\
=\lambda^{-1} C \int_{0}^{2 T} f(v(\eta))[\sinh \lambda(-T+\eta)-\sinh \lambda(-T)] d \eta .
\end{array}
$$

Addition of the above two equations gives

$$
\begin{gathered}
\int_{0}^{2 T} d \xi\left[\int_{0}^{\xi} G_{1}(\xi-\eta) f(v(\eta)) d \eta+\int_{\xi}^{2 T} G_{1}(2 T+\xi-\eta) f(v(\eta)) d \eta\right] \\
=\lambda^{-2} \int_{0}^{2 T} f(v(\eta)) d \eta .
\end{gathered}
$$

By integrating (2.4) over $(0,2 T)$ the above relation implies that $\int_{0}^{2 T} v(\xi) d \xi=0$. This shows that $v(\xi)$ satisfies all the equations in (1.1)-(1.3).

To show the converse, when $v(\xi)$ is a solution of $(1.1)-(1.3)$ we consider the function

$$
\tilde{v}(\xi)=\int_{0}^{2 T} K(\xi, \eta) f(v(\eta)) d \eta
$$

In view of the above argument, $\tilde{v}$ satisfies the equation

$$
\lambda^{2} \tilde{v}_{\xi \xi}=\tilde{v}_{\xi \xi \xi \xi}+(f(v))_{\xi \xi}
$$

and the conditions (1.2)-(1.3). Let $w=\tilde{v}-v$. Then $w$ satisfies (1.2)-(1.3) and the linear equation $\lambda^{2} w_{\xi \xi}=w_{\xi \xi \xi \xi}$. The solution of $w_{\xi \xi}$ is in the form $w_{\xi \xi}=c_{1} \cosh \lambda \xi+c_{2} \sinh \lambda \xi$. It is easily seen from the boundary condition (1.2) with $k=2,3$ that $c_{1}=c_{2}=0$ and thus $w_{\xi \xi}=0$. The condition (1.2) with $k=1$ and the relation (1.3) require that $w$ must be identically zero. Hence $\tilde{v}=v$ and is a solution of the integral equation (2.4). This completes the proof of the theorem for the case $\alpha>0$. The proof for the case $\alpha<0$ is exactly the same. 
3. Periodic traveling wave solution. It is seen from Theorem 1 that if $v(\xi)$ is a solution of the integral equation (1.4) then it can be extended to a periodic function $\bar{v}(\xi)$ on $\mathbf{R}$ such that $\bar{v}(\xi)$ is a solution of (1.1)-(1.3) and satisfies the additional relation

$$
\bar{v}(\xi)=\bar{v}(\xi+2 T) \text { for all } \xi \in \mathbf{R} .
$$

With this extension the function $u(x-\beta t) \equiv \bar{v}(\xi)$ is a periodic traveling wave solution of the Boussinesq equation (1.0). In order to establish the existence problem for (1.4) we seek a solution in the Banach space of continuous $2 T$-periodic functions $C_{2 T}(\mathbf{R})$ equipped with the maximum norm $\|\cdot\|$. Denote by $B_{r}$ the ball in $C_{2 T}(\mathbf{R})$ with center zero and radius $r>0$. Then by letting

$$
(A v)(\xi) \equiv \int_{0}^{2 T} K(\xi, \eta) f(v(\eta)) d \eta \quad \text { for } \xi \in[0,2 T]
$$

a natural extension of $(A v)(\xi)$ for $\xi \in \mathbf{R}$ ensures that $A$ maps $C_{2 T}(\mathbf{R})$ into itself. Furthermore, by letting

$$
\begin{gathered}
\bar{K}_{1}=\max \{|(\lambda T / \sinh \lambda T) \cosh \lambda(T-z)-1| ; 0 \leqslant z \leqslant 2 T\} \\
\bar{K}_{2}=\max \{|(\lambda T / \sin \lambda T) \cos \lambda(T-z)-1| ; 0 \leqslant z \leqslant 2 T\} \\
M=\max \left\{\|f(v)\| ; v \in B_{r}\right\}
\end{gathered}
$$

we have the following compactness property of the operator $A$.

THEOREM 2. For any bounded subset $S$ of $C_{2 T}(\mathbf{R})$ the operator $A: S \rightarrow C_{2 T}(\mathbf{R})$ given by (3.1) is compact on $S$. In particular, if there is a ball $B_{r}$ such that

$$
\lambda \geqslant\left(M \bar{K}_{i} / r\right)^{1 / 2} \quad(i=1,2)
$$

and $\lambda T \neq n \pi$ when $\alpha<0$ then $A$ maps $B_{r}$ into itself and the integral equation (1.4) has at least one solution in $B_{r}$.

Proof. It is easily seen from the proof of Theorem 1 that the operator $A$ can be extended to $C_{2 T}(\mathbf{R})$ such that $(A v)(\xi+2 T)=(A v)(\xi)$ for all $\xi \in \mathbf{R}$. Since $f(v)$ is bounded on any bounded subset of $C_{2 T}(\mathbf{R}),\|A v\|$ is uniformly bounded on $S$. Moreover, direct differentiation of $(A v)(\xi)$, using the property $G_{i}(0)=G_{i}(2 T), i=1,2$, gives

$$
\begin{aligned}
\frac{d}{d \xi}(A v)(\xi)=(-2 \sinh \lambda T)^{-1} & {\left[\int_{0}^{\xi} \sinh \lambda(T-\xi+\eta) f(v(\eta)) d \eta\right.} \\
& \left.+\int_{\xi}^{2 T} \sinh (-T-\xi+\eta) f(v(\eta)) d \eta\right]
\end{aligned}
$$

for $\alpha>0$; and

$$
\begin{aligned}
\frac{d}{d \xi}(A v)(\xi)=(2 \sin \lambda T)^{-1}\left[\int_{0}^{\xi} \sin \lambda(T-\xi+\eta) f(v(\eta)) d \eta\right. \\
\\
\left.\quad+\int_{\xi}^{2 T} \sin (-T-\xi+\eta) f(v(\eta)) d \eta\right]
\end{aligned}
$$


for $\alpha<0$. The above relation implies that for some constant $M_{1}$, independent of $v$,

$$
\left\|\frac{d}{d \xi}(A v)\right\| \leqslant M_{1} \quad \text { for all } v \in S .
$$

Hence $A S$ is equicontinuous. Then Arzela's theorem ensures that $A$ is compact on $S$.

To show that $A$ has a fixed point in $B_{r}$ when (3.4) holds we write

$$
\begin{aligned}
\left(A_{i} v\right)(\xi)= & \int_{0}^{\xi}\left[G_{i}(\xi-\eta)-\left(2 T \lambda^{2}\right)^{-1}\right] f(v(\eta)) d \eta \\
& +\int_{\xi}^{2 T}\left[G_{i}(2 T+\xi-\eta)-\left(2 T \lambda^{2}\right)^{-1}\right] f(v(\eta)) d \eta \quad(i=1,2)
\end{aligned}
$$

Then by (2.3), (3.2), (3.3),

$$
\begin{aligned}
& \left|\left(A_{1} v\right)(\xi)\right| \\
& \leqslant\left(2 T \lambda^{2}\right)^{-1}\left[\int_{0}^{\xi}\left|\lambda T(\sinh \lambda T)^{-1} \cosh \lambda(T-\xi+\eta)-1\right||f(v(\eta))| d \eta\right. \\
& \left.\quad+\int_{\xi}^{2 T}\left|\lambda T(\sinh \lambda T)^{-1} \cosh \lambda(-T-\xi+\eta)-1\right||f(v(\lambda))| d \eta\right] \\
& \leqslant\left(2 T \lambda^{2}\right)^{-1} \bar{K}_{1} \int_{0}^{2 T}|f(v(\eta))| d \eta \leqslant \lambda^{-2} \bar{K}_{1} M \quad\left(v \in B_{r}\right),
\end{aligned}
$$

and similarly,

$$
\left|\left(A_{2} v\right)(\xi)\right| \leqslant \lambda^{-2} \bar{K}_{2} M \quad\left(v \in B_{r}\right) .
$$

In view of (3.4), we have $\left\|A_{i} v\right\| \leqslant r$ for all $v \in B_{r}, i=1$,2. It follows from the Schauder's fixed point theorem that $A_{i}$ has a fixed point in $B_{r}$ for each $i=1,2$. Hence Eq. (1.4) has a solution for both cases $\alpha>0$ and $\alpha<0$.

An immediate consequence of Theorem 2 is the existence of a periodic traveling wave solution for the nonhomogeneous Boussinesq equation in the form

$$
u_{t t}=\alpha u_{x x x x}+\left(f_{0}(u)\right)_{x x}+h\left(x-\beta_{0} t\right)
$$

where $h$ is a $2 T$-periodic continuous function of $\xi \equiv x-\beta_{0} t$. In fact, by letting $v(\xi)=$ $u\left(x-\beta_{0} t\right)$ and choosing any $2 T$-periodic function $H(\xi)$ such that $H^{\prime \prime}(\xi)=h(\xi)$, Eq. (3.10) may be written in the form of (1.1) except with $f(v)$ given by

$$
f(v)=|\alpha|^{-1}\left[f_{0}(v)+H\right] .
$$

In view of Theorems 1 and 2 we have the following.

COROLlary. Let $\lambda T \neq n \pi$ when $\alpha<0$ and let

$$
M_{r}=\max \left\{\left\|f_{0}(v)+H\right\| ;\|v\| \leqslant r\right\} .
$$

Then for any $\beta_{0}, r$ with $\beta_{0}^{2} \geqslant M_{r} \bar{K}_{i} / r, i=1,2$, the nonhomogeneous Boussinesq equation (2.5) has a $2 T$-periodic traveling wave solution $u\left(x-\beta_{0} t\right)$ and $\left|u\left(x-\beta_{0} t\right)\right| \leqslant r$.

Proof. Since $\lambda^{2}=|\alpha|^{-1} \beta_{0}^{2}$ and $M=|\alpha|^{-1} M_{r}$ when $f(v)$ is given by (3.11), the condition $\lambda^{2} \geqslant M \bar{K}_{i} / r$ in Theorem 2 is equivalent to $\beta_{0}^{2} \geqslant M_{r} \bar{K}_{i} / r$. In view of Theorem 2 the integral equation (1.4) has a solution $v^{*} \in B_{r}$. But by Theorem 1, $v^{*}$ is also a solution of 
(1.1)-(1.3) it can be extended to a smooth periodic function $\bar{v}(\xi)$ in the space $C_{2 T}(\mathbf{R})$. Clearly $u\left(x-\beta_{0} t\right) \equiv \bar{v}(\xi)$ is a traveling wave solution of (3.10).

Theorem 2 and its corollary ensure that both the homogeneous and the nonhomogeneous Boussinesq equation have at least one periodic traveling wave solution for some suitable values of $\beta$ and $\beta_{0}$. In the case of the homogeneous equation, however, this solution may be the trivial function $v^{*}=0$. In order to obtain nontrivial solution we apply Theorem 2 by constructing a suitable subset $S$ so that $A$ maps $S$ into itself. To this end we restrict our attention to the usual Boussinesq equation $(1.1)$ where $f_{0}(u)=a u^{2}$ and $a>0$. Both the good and the bad Boussinesq equation will be considered.

By identifying the operators $A_{1}, A_{2}$ in (3.7) for the two case $\alpha>0$ and $\alpha<0$ we choose the corresponding subset in the form

$$
S_{i}=\left\{v \in C_{2 T}(\mathbf{R}) \cap C^{1}(\mathbf{R}) ;\|v\|_{2} \leqslant r_{i},\left\|v^{\prime}\right\|_{2} \leqslant r_{i}^{*}, v(0) \geqslant \delta_{i}\right\} \quad(i=1,2)(3.13)
$$

where $r_{i}, r_{i}^{*}, \delta_{i}$ are some positive constants and $\|\cdot\|_{2}$ is the usual $L_{2}$-norm. The requirement $v(0) \geqslant \delta_{i}$ excludes the zero function in $S_{i}$. Denote by $\bar{S}_{i}$ the closure of $S_{i}$ in $C_{2 T}(\mathbf{R})$. We show by choosing the constants

$$
\left.\begin{array}{l}
r_{i}=(2 T)^{1 / 2} \lambda^{2}|\alpha|\left(a \bar{K}_{i}\right)^{-1} \\
r_{i}^{*}=|\alpha| \lambda^{4}(2 T)^{3 / 2}\left(2 a \bar{K}_{i}^{2} \sigma_{i}\right)^{-1} \\
\delta_{i}=(|\alpha| / 2 a)\left(\lambda^{2}+\left[\lambda^{4}+(2 a /|\alpha|)^{2}\left(\sigma_{i} r_{i} r_{i}^{*}+(2 T)^{-1} r_{i}^{2}\right)\right]^{1 / 2}\right)
\end{array}\right\} \quad i=1,2
$$

where $\sigma_{1}=1, \sigma_{2}=|\sin \lambda T|^{-1}$, the operator $A_{i}$ given by (3.7) with $f(v)=(a /|\alpha|) v^{2}$ has a fixed point in $\bar{S}_{i}$. Specifically, we have the following existence theorem.

Theorem 3. Let $f_{0}(u)=a u^{2}$ and let $\lambda T \neq n \pi$ when $\alpha<0$. Then Eq. (1.0) has a nontrivial periodic traveling wave solution $\bar{v}(\xi) \equiv u(x-\beta t)$ and $\bar{v} \in \bar{S}_{1}$ when $\alpha>0$ and $\bar{v} \in \bar{S}_{2}$ when $\alpha<0$ whenever $\bar{S}_{i}$ is nonempty.

Proof. It is clear that $\overline{S_{i}}$ is a closed convex subset of $C_{2 T}(\mathbf{R})$. Furthermore, $\bar{S}_{i}$ is bounded in $C_{2 T}(\mathbf{R})$. For given any $v \in S_{i}$, the mean value theorem implies that for some $\xi^{*} \in[0,2 T]$,

$$
v\left(\xi^{*}\right)=(2 T)^{-1} \int_{0}^{2 T} v(\eta) d \eta
$$

Write

$$
v(\xi)=v\left(\xi^{*}\right)+\int_{\xi^{*}}^{\xi} v^{\prime}(\eta) d \eta \quad(\xi \in[0,2 T]) .
$$

Then the Schwarz inequality gives

$$
|v(\xi)| \leqslant\left|v\left(\xi^{*}\right)\right|+\int_{0}^{2 T}\left|v^{\prime}(\eta)\right| d \eta \leqslant(2 T)^{-1 / 2}\|v\|_{2}+(2 T)^{1 / 2}\left\|v^{\prime}\right\|_{2} \leqslant M_{i},
$$

where $M_{i}$ is a constant independent of $v \in S_{i}$. Hence $\|v\| \leqslant M_{i}$ for all $v \in S_{i}$ as well as for $v \in \bar{S}_{i}$. In view of Theorem 2 it suffices to show that $A \bar{S}_{i} \subset S_{i}$. 
Let $v \in S_{i}$ and let $A_{i} v$ be given by (3.7) with $f(v)=(a /|\alpha|) v^{2}$. Then by the estimate (3.8),

$$
\begin{aligned}
\left|\left(A_{i} v\right)(\xi)\right| & \leqslant\left(2 T \lambda^{2}\right)^{-1} \bar{K}_{i} \int_{0}^{2 T}(a /|\alpha|) v^{2}(\eta) d \eta \\
& =a \bar{K}_{i}\left(2 T \lambda^{2}|\alpha|\right)^{-1}\|v\|_{2}^{2} .
\end{aligned}
$$

The definition of $r_{i}$ in (3.14) implies that

$$
\left\|A_{i} v\right\|_{2} \leqslant a \bar{K}_{i}\left(2 T \lambda^{2}|\alpha|\right)^{-1}(2 T)^{1 / 2} r_{i}^{2}=r_{i} .
$$

Moreover, by letting $\xi=0$ in (3.7), performing integration by parts and using the periodic property of $v(\xi)$ we have

$$
\begin{aligned}
\left(A_{1} v\right)(0)= & (a /|\alpha|) \int_{0}^{2 T}\left[C \cosh \lambda(-T+\eta)-\left(2 T \lambda^{2}\right)^{-1}\right] v^{2}(\eta) d \eta \\
= & (a C / \lambda|\alpha|)\left[2 \sinh \lambda T v^{2}(0)-2 \int_{0}^{2 T} \sinh \lambda(-T+\eta) v(\eta) v^{\prime}(\eta) d \eta\right] \\
& -a\left(2 T \lambda^{2}|\alpha|\right)^{-1}\|v\|_{2}^{2}
\end{aligned}
$$

where $C=(2 \lambda \sinh \lambda T)^{-1}$. Since $v(0) \geqslant \delta_{1}$ for $v \in S_{1}$ and $|\sinh (T-z)| \leqslant \sinh \lambda T$ for $z \in[0,2 T]$ the above relation implies that

$$
\left(A_{1} v\right)(0) \geqslant\left(a / \lambda^{2}|\alpha|\right) \delta_{1}^{2}-\left(a / \lambda^{2}|\alpha|\right) r_{1} r_{1}^{*}-a\left(2 T \lambda^{2}|\alpha|\right)^{-1} r_{1}^{2} .
$$

It follows from the choice of $\delta_{1}$ in (3.14) that $\left(A_{1} v\right)(0) \geqslant \delta_{1}$. A similar argument shows that $\left(A_{2} v\right)(0) \geqslant \delta_{2}$. Since by the relations (3.5)-(3.6),

$$
\begin{aligned}
&\left|\frac{d}{d \xi}\left(A_{1} v\right)(\xi)\right| \leqslant(1 / 2)\left[\int_{0}^{\xi}|f(v(\eta))| d \eta+\int_{\xi}^{2 T}|f(v(\eta))| d \eta\right] \\
& \leqslant(a / 2|\alpha|)\|v\|_{2}^{2}, \\
&\left|\frac{d}{d \xi}\left(A_{2} v\right)(\xi)\right| \leqslant a(2|\alpha||\sin \lambda T|)^{-1}\|v\|_{2}^{2} .
\end{aligned}
$$

We see that $\left\|\left(A_{i} v\right)^{\prime}\right\| \leqslant a\left(2|\alpha| \sigma_{i}\right)^{-1}(2 T)^{1 / 2} r_{i}^{2}=r_{i}^{*}$. This shows that $A_{i} v \in S_{i}$ for every $v \in S_{i}$. Finally, for $v \in \bar{S}_{i}$ there is a sequence $\left\{v_{n}\right\}$ in $S_{i}$ such that $v_{n} \rightarrow v$ in $C_{2 T}(\mathbf{R})$. In view of (3.5)-(3.7), $A_{i} v_{n} \rightarrow A_{i} v$ and $\left(A_{i} v_{n}\right)^{\prime} \rightarrow(A v)^{\prime}$ in $C_{2 T}(\mathbf{R})$ as $n \rightarrow \infty$. It follows from the uniform convergence of these sequences that $\left\|A_{i} v\right\|_{2} \leqslant r_{i},\left(A_{i} v\right)(0) \geqslant \delta_{i}, A v \in C^{1}(\mathbf{R})$ and $\left\|(A v)^{\prime}\right\|_{2} \leqslant r_{i}^{*}$. This proves that $A_{i} \bar{S}_{i} \subset S_{i}$. By Theorem 2 and the Schauder's fixed point theorem, $A_{i}$ has a fixed point $v \in \bar{S}_{i}$. The extension $\bar{v}(\xi)$ of $v(\xi)$ for $\xi \in \mathbf{R}$ leads to a periodic traveling wave solution $\bar{v}(\xi) \equiv u(x-\beta t)$. This completes the proof of the theorem.

The result of Theorem 3 illustrates that when $f_{0}(u)=a u^{2}$, no matter how small the constant $a$ may be, both the good and the bad Boussinesq equation has a periodic traveling wave solution of period $2 T$ for any fixed period $2 T$ (except with the restriction that $T \neq n \pi / \lambda$ when $\alpha<0$ ). However, this is no longer the case when $a=0$. In the case of $\alpha<0$, for example, the general solution to (1.1) corresponding $f_{0}(u)=0$ is given by

$$
v(\xi)=B_{1} \cos \lambda \xi+B_{2} \sin \lambda \xi+B_{3} \xi+B_{4}
$$


where $B_{i}, i=1, \ldots, 4$, are arbitrary constants. It is easily seen that $v(\xi)$ is periodic solution of (1.1)-(1.3) only if $\lambda T=n \pi$ and $B_{3}=B_{4}=0$. This is in sharp contrast to the case $a \neq 0$.

\section{REFERENCES}

[1] T. B. Benjamin, Lectures on nonlinear wave motion, 3-48, Nonlinear Wave Motion (ed. Alan C. Newell), Vol. 15, Lectures in Applied Mathematics

[2] P. L. Bhatnagar, Nonlinear waves in one-dimensional dispersive systems, Clarendon Press, Oxford, 1979

[3] R. Hirota, Exact $N$-soliton solution of the wave equation of long waves in shallow-water and in nonlinear lattices, J. Math. Phys. 14, 810-814 (1973)

[4] H. P. Mckean, Boussinesq's equation on the circle, Comm. Pure Appl. Math. 34, 599-691 (1981)

[5] P. Prasad and R. Ravindran, $A$ theory of nonlinear waves in multi-dimensions: with special reference to surface waves, J. Inst. Math. and its Appl. 20, 9-20, (1977)

[6] M. Toda, and M. Wadati, A soliton and two solitons in an exponential lattice and related equations, J. Phys. Soc. Japan 34, 18-25, (1973)

[7] N. J. Zabusky, A synergetic approach to problems of nonlinear dispersive wave propagation and interaction, 223-258, Nonlinear Partial Differential Equations (ed. by W. F. Ames), Academic Press, New York, 1967 